\title{
Differenz
}

Revista internacional de estudios heideggerianos y sus derivas contemporáneas

AÑO 7, NÚMERO 6: JULIO DE 2020. ISSN 2386-4877 - DOI: 10.12795/Differenz.2020.i06.02

[pp. 31-52]

Recibido: 06/06/2020

Aceptado: 19/06/2020

\section{El nihilismo como ausencia de fines y su superación en el pensar histórico del Ser.}

\section{Nihilism as the absence of goals and its overcoming in be-ing historical thinking.}

\author{
César Gómez Algarra \\ Université Laval/Universidad de Valencia
}

Resumen:

La publicación, desde 1989, de los tratados póstumos sobre la historia del Ser profundiza el sentido del problema del nihilismo en la filosofía de Heidegger. En el presente artículo buscamos demostrar que estos textos (de las Contribuciones a la filosofía a los Cuadernos negros) permiten una nueva perspectiva interpretativa del pensar histórico del Ser: la de ausencia de fines (Zielen) y la búsqueda como fin en sí mismo. Esto nos ayuda a entender mejor tanto el problema del nihilismo en Heidegger, como la forma especial que adopta su solución: la superación del nihilismo en el pensar del Ereignis.

Palabras clave: Heidegger; Nihilismo; Ereignis; Historia del Ser. 


\section{Abstract:}

Since 1989, the publication of the posthumous works of Heidegger has been deepening the meaning of nihilism in his philosophy. In this paper, we will argue that these texts, from the Contributions to Philosophy to the Black Notebooks, enable a new perspective to interpret his project of be-ing historical Thinking: namely the absence of goals (Zielen), and the search as the goal itself. This allows us to fully grasp the problem of nihilism in Heidegger's thought, as well as his solution to it: the overcoming of nihilism through the Ereignis-denken.

Keywords: Heidegger; Nihilism; Ereignis; History of Be-ing.

\section{Introducción.}

Nihilismo: "falta" el fin; "falta" la respuesta al "¿por qué?". F. Nietzsche. Fragmentos póstumos, 9 (35), Otoño 1887.

A partir de los años 30, Heidegger caracteriza la modernidad a través de una serie de conceptos diversos y plurales que forman, pese a su multiplicidad, una constelación coherente. Esta constelación adopta una forma particular y muy diferenciada en los textos póstumos ${ }^{1}$, movilizando y ordenando en un nuevo marco la dominación de la realidad efectiva (Wirklichkeit) a partir de la maquinación o fabricación (Machenschaft), en consonancia con la primacía del hacer y de la vivencia (Erlebnis). Sin embargo, todos estos fenómenos confluyen en uno, como subrayará Heidegger en el tomo 67, Metaphysik und Nihilismus, y éste no es otro que el olvido del ser como su esencia más íntima y, por tanto, según la interpretación histórica del filósofo alemán, olvido que no es sino la metafísica misma y que puede ser nombrado también como nihilismo².

Se podría defender y justificar que, en la medida en que el nihilismo es la esencia y el destino originario de la metafísica, todo el conjunto del pensar del Ereignis en estos años está dirigido contra él. En el marco de este trabajo, sin embargo, queremos presentar

1 Con ello referimos a los tratados de la historia del Ser, del volumen 65 al 73 de la Gesamtausgabe, a los Cuadernos negros (94 a 96 en particular) y el volumen 82 de comentarios a las obras publicadas en vida. Para una presentación en castellano, clara y sucinta, de los tratados póstumos, remitimos a XolocotzI, Á.; Gibu, R.; HUerTA, V.; VerAZA, P. Heidegger. Del sentido a la historia. México D. F. Plaza y Valdés, 2014. pp. 90-112.

2 HeIdegGer, M. Metaphysik und Nihilismus. GA 67. Frankfurt am Main, Klostermann, 1999, p. 48. 
y analizar una de las vías de acceso al problema que ha recibido menos atención. Pese a ello, concierne varios temas clave del pensar histórico del Ser contenido en las Contribuciones a la filosofía y demás textos contemporáneos, y permite arrojar nueva luz sobre el conjunto del proyecto que allí se lleva a cabo. Se trata de la relación esencial entre nihilismo y ausencia de fines, y la posibilidad de superar tal relación a través de la idea de la búsqueda del $\mathrm{Ser}^{3}$. Por la forma más pulida y rigurosa que alcanzan estas ideas en los volúmenes 65 y 66, Contribuciones y Meditación, será en estas dos obras en las que nos centraremos, pero queremos subrayar aquí que la cuestión abunda en todos los textos esotéricos ${ }^{4}$, adoptando formulaciones muy variadas, incluso repetitivas a veces.

En primer lugar, expondremos la caracterización del nihilismo en los tratados póstumos como época del abandono del Ser, ocultado por la fabricación ${ }^{5}$ y la efectividad. Habiendo presentado este punto, insistiremos en la manera en que la ausencia de fines (Zielen) y de respuesta a la pregunta por el "¿por qué?", definición clásica del nihilismo, determinan la historia dominada por la objetivación y la primacía del ente. Esto constituirá nuestra primera parte. La segunda parte procurará ahondar en las respuestas que el pensar de la historia del Ser ofrece a la encrucijada nihilista. Se tratará entonces de desplegar un pensar más allá de fines, que debe ser entendido como búsqueda constante del Ser. Tal búsqueda, en tanto que superación al problema del nihilismo, no será comprensible del todo sin explicar el papel que puede jugar el ser humano, ya no como centro determinante de las relaciones con el ser y con el ente, sino como Da-sein, y es su nuevo rol como buscador del Ser que intentaremos elucidar en la conclusión.

3 Como es ya habitual, escribimos "Ser" con mayúscula para referirnos al Seyn alemán, y "ser" con minúscula para Sein, ser del ente.

4 Para la caracterización esotérica de los tratados sobre la historia del Ser, opuestos a los cursos exotéricos, véase especialmente SOMMeR, C. "Für die Wenigen- Für die Seltenen. Doctrine ésotérique et art d'écrire chez Heidegger", en SCHNELL, A. (Ed.). Lire les "Beiträge zur Philosophie" de Heidegger. París, Hermann, 2017, pp. 7-24 y TRAWnY, P. Adyton. Heideggers esoterische Philosophie. Berlin, M\&S, 2010.

5 Nos inspiramos de las indicaciones de A. Boutot en la presentación a su traducción francesa de Besinnung (Méditation. París, Gallimard, 2019, pp. 19-20). Preferimos el término "fabricación" frente al habitual de "maquinación" o "maniobra" por dos razones: 1) El propio Heidegger se distancia del sentido de intriga y acción humana, como algo derivado, en el $\S 61$ de las Contribuciones y el $\S 42$ de La historia del Ser, y 2) El uso de "fabricación" conecta bien con lo esencial de la Machenschaft, la primacía del hacer (faber), pero sobre todo un más allá del hacer humano, en sus despliegues calculadores y técnicos. 


\section{Breve caracterización del nihilismo según la historia del Ser.}

\subsection{El abandono del ser y la fabricación como nihilismo.}

Como destaca Heidegger en las Contribuciones a la filosofía, la experiencia fundamental en la que se ve inmerso el ser humano en la época del final de la Modernidad, es que el ser abandona al ente, y que, por lo tanto, la palabra y el sentido mismo de ser se han evaporado. Este fenómeno "epocal" es caracterizado a través de dos conceptos de primer orden, abandono y olvido del ser (Seinsverlassenheit y Seinsvergessenheit) que, conectando con el proyecto de Ser y tiempo, aportan una dimensión y una comprensión histórica al problema mismo, que se interrogaba, al menos aparentemente, en un marco trascendental en el libro de 1927. Ya no se trata de plantear una ontología fundamental capaz de ofrecer respuestas definitivas al problema del sentido del ser, sino que el problema, propiamente dicho, es insertado en el desarrollo de la historia misma de la filosofía, desde Platón hasta Nietzsche.

En respuesta a esta situación de abandono, los modernos sufren un profundo desarraigo y una tribulación (Not) que, sin embargo, es difícil aprehender como tal, incluso simplemente percibirla. Más concretamente, tan sólo algunos de entre ellos, los que no se ven subyugados por la dominación del abandono del ser en el ente, se revelarán capaces de captar este desmoronamiento de sentido. La mayoría, los más numerosos, son más bien los que, embaucados por este fenómeno, se hunden en lo que Heidegger caracteriza como primacía de las vivencias (Erlebnisse), en la que todo es medido a partir de la experiencia y del valor superficialmente subjetivo, experiencia mediatizada y dirigida por la fabricación moderna. Una primera consecuencia de la situación moderna y nihilista es entonces la de un tipo de huida o de refugio para hacer frente al abandono del ser, huida definida como ocultamiento del abandono del ser "en la creciente validez del cálculo, de la velocidad y de la exigencia de lo masivo"6. La caracterización del ocultamiento a través de estos fenómenos subraya, incidentalmente, el papel negativo que juega la ciencia experimental y el progreso técnico en los textos de estos años ${ }^{7}$, anunciando ya las tematizaciones sobre el Gestell de los años de posguerra.

Frente a la mayoría, están precisamente los pocos (die Wenigen), aquellos que experimentan con mayor profundidad la tribulación que supone sentir que el Ser ha

6 HeIDEgGeR, M. Beiträge zur Philosophie (Vom Ereignis). GA 65. Frankfurt am Main, Klostermann, 1989, p. 120. Citamos las traducciones disponibles en español, modificándolas si es pertinente y sin previo aviso para una mejor comprensión del argumento expuesto. Las referencias se encuentran en la bibliografía.

7 A este respecto, son especialmente importantes los $\S \S 73-80$ de las Contribuciones, junto a la segunda parte del volumen 76 de la Gesamtausgabe, dedicada a la génesis de la ciencia moderna. 
dejado de lado al ente, y que vivimos entonces atrapados por lo que es (en un sentido determinado y limitado, in-esencial (unwesentlich), que diría Heidegger), como lo que está dado ahí, presente y efectivo (wirklich). Heidegger insiste en numerosos apartados que esta situación, dominada por la fabricación, supone e implica una especie de estancamiento, como una época de repetición y continuación indefinida del estado de cosas existente. A través de ella, el ser humano se encuentra condenado a una "servidumbre incondicionada", de la que solo puede salir a través de un combate o lucha (Kampf): sin una experiencia auténtica del Ser en cuanto tal, ninguna decisión verdadera capaz de un cambio auténtico es posible ${ }^{8}$.

En el marco conceptual moderno, presidido por el cálculo, la medida y la experiencia reducida de las vivencias subjetivas, las decisiones no dejan de moverse dentro de las posibilidades ya determinadas por la metafísica y, por tanto, dentro de la esencia del nihilismo. Sin embargo, los pocos, atentos al Ser, deben revelarse capaces no sólo de experimentar el desarraigo, sino de reconducirlo hacia una decisión auténtica, una que esté enraizada en la necesidad de la tribulación (Notwendigkeit der Not) y, por lo tanto, en correlación con el Ser como tal. Es justamente a través de la tribulación, y de las tonalidades fundamentales que con ella se despiertan, que el ser humano se ve decidido a interrogar al Ser como lo sumamente cuestionable, lo que es más digno de ser cuestionado (fragwürdig). Tal decisión no es, simple y llanamente, el acto de una voluntad decidida, lo que la situaría aún en la comprensión moderna de la decisión como acción consciente de un sujeto. Consiste más bien en la decisión de una posibilidad radical, una posibilidad entre "historia o pérdida de historia", es decir, entre la "pertenencia al Ser o el abandono en el no ente (Unseienden)" en el que se consume la fabricación y el dominio de lo efectivo'.

Profundicemos este punto. La dificultad a la que se enfrentan los pocos es que deben ser capaces de pensar fuera del esquema metafísico, un esquema que reduce a ente todo lo que se tiene delante, y que, a través las distintas determinaciones históricas de la relación con el ser (Sein), culmina en su manifestación moderna como relación sujeto-objeto. Pero esta culminación no es más que el cumplimiento de un proceso que empezó con Platón y que caracteriza fundamentalmente a la metafísica como nihilismo: la primacía de la presencia y la constancia (oúsia; Beständigkeit) conlleva indefectiblemente el descrédito a todo lo que no sea según estos parámetros. Cabe destacar aquí, respecto al problema del nihilismo, una de las claves más importantes a la hora de determinar el enfrentamiento

8 HeIdegGer, M. Metaphysik und Nihilismus. Op. cit., p. 147.

9 HeIDEgGeR, M. Beiträge zur Philosophie (Vom Ereignis). Op. cit., p. 100. 
heideggeriano con la historia de la metafísica: la imposibilidad de pensar la nada. En efecto, ya anunciado en la conferencia inaugural de 1929 sobre ¿Qué es metafísica?, es uno de los temas omnipresentes en los textos de los años 30, y alcanza su mayor riqueza conceptual en el comentario a Hegel sobre la negatividad, primera parte del volumen 68 de la Gesamtausgabe. El nihilismo metafísico es, en gran medida, y en consonancia con el dominio de la fabricación, la imposibilidad de ver más allá de lo efectivo, y por tanto la radicalización de una tendencia que, de manera general, ya se encontraba in nuce en la filosofía occidental (no tanto desde Parménides quizá, como desde El sofista de Platón): el tratamiento de la nada como no-ente, y en consecuencia la prohibición de pensar la nada a partir de ella misma ${ }^{10}$. En ese sentido, si el nihilismo es esencialmente abandono del Ser, Heidegger subraya que éste no es tan sólo caracterizado por la evaporación de todo lidiar con el Ser en cuanto tal, sino también con la nada como tal. Así pues, el pensar histórico del Ser pretende superar este problema considerando y enfrentándose a la nada misma, sin paliativos dialécticos y sin recursos hegelianos, falsas soluciones de la culminación de la metafísica.

Habiendo expuesto esta caracterización general de la comprensión del nihilismo que los textos del pensar histórico del Ser presentan, podemos, en un segundo momento, entrar más en detalle en el punto decisivo al que Heidegger se enfrenta y que aquí nos interesa: la figura que adopta el nihilismo en la época moderna como ausencia de fines.

\subsection{La ausencia de fines y la pregunta "¿por qué?".}

Para Heidegger, cuando el esquema metafísico de la fabricación, (esquema que solo tiene en cuenta lo que es en la medida en que se da efectivamente ahí y produce efectos calculables), es aplicado a las nociones de decisión, causas y fines, constituye un obstáculo difícilmente franqueable, y un signo preponderante del nihilismo. Es éste uno de los problemas en los que el pensar histórico del Ser recurre o, por así decirlo, llama a Nietzsche al estrado como testigo excepcional para confrontarse a su tratamiento del problema ${ }^{11}$.

10 Por lo tanto, y a pesar de su innegable cercanía, la tentativa de E. Severino, expuesta magistralmente en Esencia del nihilismo, puede caracterizarse como un proyecto invertido y opuesto al del pensar histórico del Ser en Heidegger.

11 Otro punto de primera importancia sería la crítica heideggeriana a la noción de cría (Züchtung) del superhombre en Nietzsche como último avatar del humanismo metafísico y del tratamiento del ser humano como ente vorhanden. A esta figura nietzscheana de la fabricación, Heidegger opondría partir de la preparación (Vorbereitung) del pensar del Ser. Esperamos poder tratar este problema en un próximo trabajo. 
Encontramos una explicación más precisa de la cuestión en el §72 de las Contribuciones, con el oportuno título de "El nihilismo".

Nietzsche, afirma Heidegger, ha comprendido el nihilismo como la desvalorización de todos los valores supremos, como la pérdida y ausencia de toda meta o fin, reivindicando una recuperación de éstos a partir de un nuevo prisma. La tarea del filósofo del futuro es entonces la creación y la posición de nuevos valores, alejados de la voluntad de nada que el cristianismo extendió. Nótese, además, que Heidegger reconoce en este proyecto nietzscheano mucho más que un diagnóstico de "psicología cultural", subrayando la mala comprensión del problema del nihilismo por parte de sus contemporáneos, que son incapaces de situar a Nietzsche en su lugar correspondiente. Es decir, como continuación y culminación de la tradición metafísica ${ }^{12}$. Sin embargo, el propio Nietzsche no habría logrado alcanzar la claridad necesaria del fenómeno en su enfrentamiento con la esencia del nihilismo. Sabemos que, para el filósofo de Friburgo, la manera nietzscheana de plantear el problema supone ya de entrada una recaída en cierto platonismo vulgar, y numerosos son los apartados en los que Heidegger critica el intento de inversión del platonismo como siendo tan sólo una apariencia de superación de la metafísica, ya sea en la concepción de la verdad-apariencia como en la aceptación dionisíaca del devenir. En la medida en que toda inversión pretende ser un enfrentamiento con aquello que aspira a dejar atrás y queda insertado en su desarrollo e historia, un movimiento del pensar semejante jamás podrá alcanzar lo esencial ${ }^{13}$.

Sin querer discutir aquí la pertinencia de esta interpretación, que ha sido muy criticada, lo que nos interesa destacar es cómo en las Contribuciones se insiste sobre el pensar respecto a fines, télos, como eminentemente metafísico. Si el nihilismo implica el hundimiento del pensar en esquemas de causa-fin donde el ente domina como efectividad, donde la causa solo interesa en la medida en que es efectiva y capaz de producir un efecto, la historia del Ser debe representar la vía abierta a un nuevo tipo de pensar que logre no repetir este esquema. A fin de cuentas, Heidegger parece estar de acuerdo con Nietzsche en que el nihilismo se caracteriza principalmente por la ausencia de fines. Es más bien respecto a cómo debe el pensamiento enfrentarse a ello, o más bien cómo cree Nietzsche haber superado el problema (por la instauración de nuevos valores, a través de la voluntad de poder, que no conduzcan a la voluntad de nada, etc.), que se opera una ruptura.

12 HeIdegger, M. Beiträge zur Philosophie (Vom Ereignis). Op. cit., p. 139.

13 HeIDEGgER, M. Besinnung. GA 66. Frankfurt am Main, Klostermann, 1997, p. 385. 
A este respecto es ilustrativo el ejemplo de crítica al pensar en términos de fines presentado en el $§ 72$, sobre la confusión imperante en la época que pone al pueblo (Volk), como fin absoluto de todo proyecto y decisión. Para Heidegger, esa posición de objetivos funciona por medio de la reducción a un ente, tratando como tal y como medio en vistas a su realización efectiva lo que en ningún caso debe elevarse a tal posición. En el caso del pueblo el problema es denunciado en varias ocasiones, como el §196, llegando al extremo de considerar que

El pueblo nunca puede ser fin y objetivo, y que tal opinar sólo es una ampliación "popular" (völkisch) del pensamiento "liberal" del "yo" y de la representación económica de la conservación (Erhaltung) de la vida ${ }^{14}$.

En el pensar histórico del Ser se trata justamente de lo contrario, de entender al pueblo como un nuevo tipo de condición de posibilidad en vistas a un más allá de la comprensión metafísica. El ejemplo del pueblo es sólo uno entre muchos otros, y de igual manera pueden pensarse otros fines políticos, o la raza ${ }^{15}$, el Estado, la vida e incluso el ser humano en cuanto tal, en tanto que fin en sí mismo de sí mismo ${ }^{16}$. Todo ello apunta a una determinación esencial del nihilismo: una ausencia de fines que se convierte, paradójicamente, en una obsesión por la creación, posición y persecución de fines diversos y heterogéneos. Tal diversidad no es, además, más que un mero espejismo o ilusión, y todo esto se reúne en una importante dimensión de la repetición constante del final de la época moderna según Heidegger, donde "la época deviene cierta de haber encontrado en la vida misma 'el' fin y con ello su propia eternidad, en la que ser y devenir se hacen equiparar e intercambiar..." ${ }^{17}$. Es decir, que los fines están entrelazados en su constante fabricación, en la que nosotros nos perdemos, sin que por ello lleguemos a

14 HeIDegGer, M. Beiträge zur Philosophie (Vom Ereignis). Op. cit., p. 319. Este punto es el que abre el problema de la metapolítica en Heidegger, reveladora tanto de la cercanía con el nazismo como de su progresivo alejamiento. No pudiendo tratar este problema detalladamente en el marco de la presente investigación, remitimos a la crítica al colectivismo como fin en el $\S 47$ de Meditación, al artículo de Xolocotzı, Á., "De la metafísica del Dasein a la metapolítica del pueblo histórico. Aspectos del giro político de Heidegger", en O que nos faz pensar, 36, 2015, pp. 7-25. y al imprescindible libro de SOMMER, C. Mythologie de l'événement. Heidegger avec Hölderlin. París, Presses Universitaires de France, 2017.

15 Para un análisis crítico y original de la raza, véase AlvARADO, A., "Consideraciones sobre el cuerpo, la raza y el mestizaje a la luz de los Cuadernos negros de Heidegger", en Eikasia, 91, 2020, pp. 37-58. 16 HEIDEGGER, M. Besinnung. Op. cit., p. 234.

17 Ib. p. 285. 
rozar tan siquiera lo esencial: al Ser en cuanto tal, como Ereignis. Ésta es, sin lugar a dudas, una de las manifestaciones modernas del nihilismo ${ }^{18}$.

Se constituye así una búsqueda incesante de fines en la que Heidegger ve entremezclados fenómenos tan dispares como los bienes culturales, el cristianismo, el judaísmo e incluso el americanismo y el bolchevismo: un batiburrillo donde todo acaba confundido en lo indiferente, punto que ha sido duramente cuestionado ${ }^{19}$. En cualquier caso, respecto a esta cuestión, el filósofo alemán insiste: mientras nos movamos en el ámbito del preguntar metafísico, no se podrá evitar el pensar en términos de fines, siendo su ocasional empleo en las Contribuciones nada más que una indicación provisional y tácitamente errada. Debemos preguntarnos entonces qué puede ser un pensar ajeno al télos, un pensar que ha superado auténticamente el nihilismo. Lo que parece innegable para Heidegger es que, si no superamos este pensar metafísico, seguiremos encerrados en el reino del nihilismo que supone la dominación de la fabricación. A este respecto, al final del apartado "Ser y devenir" de Meditación, se expone, un tanto enigmáticamente, el impasse al que se somete toda filosofía que sigue creyendo ingenuamente en fines:

¿Cómo lo que antes se ha convertido a sí mismo en fin de sí mismo y toma todos los planteos de objetivo (Zwecksetzungen) al servicio de este fin, debe poder aún alguna vez preguntar por un fin, sin introducir con ello la autoaniquilación (Selbstvernichtung)? ${ }^{20}$

Esta situación, que caracteriza a la metafísica, señala a la vez los límites de su planteamiento y la necesidad de salir de su esquema de preguntas-respuestas. Según Heidegger, la cuestión es tan antigua como la historia de la filosofía misma. Es decir, el conjunto de problemas que conciernen causas y fines y su tratamiento de éstos a partir de la óptica del ente está enraizado en la determinación inicial de la metafísica respecto a la pregunta por el ¿por qué?, síntoma también del nihilismo, como ya anunciaba Nietzsche. El tratamiento más completo de esta dificultad lo encontramos en la sección XXI de Meditación, que cabe exponer a continuación, y que funciona precisamente como un tipo de preguntar transitorio hacia el otro pensar.

Dentro del proyecto de una historia del Ser, Heidegger interpreta la pregunta por el "¿por qué?" (Warum?), en su sentido metafísico, a través de dos fenómenos de primer orden:

18 HeidegGer, M. Die Geschichte des Seyns. GA 69. Frankfurt am Main, Klostermann, 1999, pp. 183-184.

19 Véase, por ejemplo, la interesante crítica a la concepción heideggeriana del liberalismo que encontramos en Polt, R. The Emergency of Being. Ithaca, Cornell University Press, 2006, pp. 227236.

20 HeIDEgGer, M. Besinnung. Op. cit., p. 285. 
la primacía del "qué" (Was?) y el asombro (thaumazein) como tonalidad fundamental del comienzo griego de la filosofía. Según esta óptica, para el filósofo alemán el preguntar por el ¿por qué? se comprendió inmediatamente en una pregunta por las causas, es decir, en una pregunta que ya planteaba lo que había que interrogar en términos de causa, objeto, y de esta manera ya reducía el ser al ente. Más concretamente, la pregunta metafísica del ¿por qué? implica, de manera velada, la desfiguración de una pregunta originaria, al pasar a la determinación del qué del ente, en la medida en que tal planteamiento es el que se considera como respuesta válida al preguntar así formulado. Así, en el fondo, esta pregunta metafísica, como pregunta conductora (Leitfrage) ya ha decidido sobre la esencia de lo que está siendo interrogado, reduciendo todo intento de respuesta al "camino de la explicación a partir de causas o de condiciones de la representatividad del ente predeterminado como objeto"21. En este sentido, tal perspectiva es coherente con la interpretación heideggeriana de la historia de la metafísica como primer comienzo: la primera experiencia con el ser se planteó como asombro, como el maravillarse ante las cosas que son, e instauró el ser del ente, su entidad, como hilo conductor de todo preguntar.

Cabe destacar que este comienzo es ya, en gran medida, lo que dio origen esencial al nihilismo y a su desarrollo en la metafísica. Heidegger apunta en estas páginas unas consideraciones sobre el asombro que completan lo que ya exponía en las Contribuciones ${ }^{22}$. Aquí debemos insistir en que, para el pensador de la Selva Negra, la tematización del asombro como tonalidad fundamental estaba, ya de antemano, condenada a un progresivo agotamiento hasta llegar a su extenuación como tal. ¿A qué se debe esto? La pregunta conductora parte del asombro, pero éste es reconducido a través de su fijación en el ente, $y$, "vencido por el creciente conocimiento del ente, cede a éste y renuncia a sí mismo..."23. De esta manera, para Heidegger la metafísica nació como progresivo tratar con el ente, como un cierto conocimiento, mientras que el fundamento, que no era otro que el Ser y su primera interrogación despertada por los griegos, permaneció decisivamente oculto y enterrado para la consecución de la historia de la filosofía, que se irá alejando más y más de la posibilidad de pensar el Ser, abandonándolo hasta convertirse definitivamente en nihilismo.

Sin embargo, la pregunta por el ¿por qué? puede entenderse de otra manera, más radical y originaria. Como pregunta de transición, ésta puede constituirse en una vía de acceso para desplegar la pregunta fundamental (Grundfrage), y a través de ella,

21 lb. p. 272.

22 Heidegger, M. Beiträge zur Philosophie (Vom Ereignis). Op. cit., p. 16, 46.

23 HeIDEgGeR, M. Besinnung. Op. cit., p. 273. 
aproximarnos al pensar del Ser como respuesta al nihilismo, punto que constituye nuestra segunda parte.

\section{Superación del nihilismo metafísico en el pensar histórico del Ser.}

\subsection{El pensar del otro comienzo como búsqueda del Ser.}

En el mismo apartado de Meditación, Heidegger insiste en que habría dos maneras de entender la pregunta por el ¿por qué?: la metafísico-nihilista que, ligada al primer comienzo determinado por el asombro, justamente ha pre-determinado toda respuesta posible a través del esquema del ente, del objeto y de la causalidad. Esta interpretación, como ya hemos dicho, culmina en la filosofía como saber sobre el ente y el correspondiente abandono del Ser en la época moderna como fabricación. La segunda interpretación pretende, al contrario, reconducirnos a una experiencia originaria del Ser. En otras palabras, con la pregunta del ¿por qué? se abre lo más inquietante y enigmático, así como lo que revela la mayor dignidad del preguntar. ¿Por qué? ya no indica la posibilidad de una respuesta siempre realizable y explicable, sino más bien, una situación en la que el ser humano ya no es poseedor de lo hecho. Esta interpretación ya está marcada, además, contra el asombro griego, por otra tonalidad fundamental: la del espanto y desposesión (Entsetzung) frente al esenciarse del Ser, tonalidad que justamente "excede todo asombro y nada común tiene con lo meramente terrible del sentir habitual"24.

Las nuevas tonalidades fundamentales constituyen uno de los puntos determinantes del intento heideggeriano de elaborar un pensar histórico del Ser en los años 30, como un pensar que se despliegue a partir o desde el Ser mismo, conservando toda la ambigüedad de una formulación semejante ${ }^{25}$. Antes que nada, debemos subrayar que, si bien en las Contribuciones hay una multitud de tonalidades fundamentales, y al principio de la obra se presentan especialmente el horror (Erschrecken), la retención (Verhaltenheit) ${ }^{26}$ y el temor respetuoso $(\mathrm{Scheu})^{27}$, aquí queremos destacar la primera, que resonando junto al espanto como des-posesión (Ent-setzung) es la que mejor permite entender la cuestión que aquí

24 Ib. p. 274.

25 DASTUR, F. "Le tournant de l'Ereignis et la pensée à venir", en SCHNELL, A. (Ed.). Op. cit., 2017, p. 149.

26 Como subraya, entre otros, Á. Xolocotzi, Heidegger da a entender en varios momentos que la retención es la tonalidad fundamental que engloba las otras tonalidades del pensar histórico del Ser. Sin embargo, el carácter esotérico y provisional de estos textos invita, a nuestro entender, a llevar a cabo una investigación completa, a riesgo de matizar este punto. Véase XoLocotzı, Á. Fundamento y abismo. Aproximaciones al Heidegger tardío. México D. F., Porrúa, 2011, pp. 71-84.

27 HeIdegger, M. Beiträge zur Philosophie (Vom Ereignis). Op. cit., pp. 11-18. 
nos ocupa. En este sentido, nos adscribimos plenamente a la interpretación que Reiner Schürmann lleva a cabo en el último capítulo de su monumental Des hégémonies brisées, priorizando en su lectura de las Contribuciones el sentido de esta tonalidad fundamental ${ }^{28}$. Más allá de la orientación que tiene la retoma particular de Heidegger en el marco de su muy personal analítica de los principios, resulta innegable que la desposesión, como bien indica su nombre, es la tonalidad ajena y opuesta a todo lo que concierne cualquier posición (Setzung), y por lo tanto a cualquier posición de valores, fines e ideas ${ }^{29}$. En ese sentido, no es irrelevante para enfrentarse a la sobreabundancia de posiciones propia del nihilismo metafísico. En el marco de este artículo, sin embargo, nos limitaremos a usarla como guía para adentrarnos en la caracterización del otro comienzo como pensar del Ser frente al nihilismo.

En este sentido, la desposesión permite una primera indicación respecto a cuál es el sentido de la pregunta fundamental u originaria. La pregunta del ¿por qué?, nos dice Heidegger, no debe ser respondida por una explicación objetiva o causal; no debe convertirse en el irremediable camino hacia una causa suprema. Según las palabras del propio Heidegger: "En la historia del otro comienzo tan sólo el Ser mismo determina la esencia del fundamento y excluye la pregunta-por qué como insuficiente" ${ }^{30}$. Si dejamos de lado la óptica metafísica de objetivación y limitación como causalidad o fundamento, la respuesta ya no será entonces la "tranquilizadora" explicación a partir del ente: debemos más bien detenernos en el esenciarse del Ser mismo y permanecer ahí, insistiendo como ahí, punto que trataremos más adelante.

Ahora bien, si esta exclusión del ¿por qué? es insuficiente, es porque se revela incapaz de abrir el verdadero ámbito de la pregunta propia del otro comienzo. Un punto que resulta particularmente interesante es cómo, en el intento de alcanzar un nuevo comienzo, Heidegger no puede, sin más, hacer tabula rasa y dejar completamente de lado todos los conceptos metafísicos con y en los que él mismo se ha formado. Subrayemos además que esta incapacidad, asumida y reconocida por momentos, estaría fundada en la comprensión del otro comienzo como una retoma, una rememoración y una rehabilitación de lo que, dentro del primer comienzo y lo que la metafísica del ente desarrolló, se quedó necesariamente oculto. Este método, que luego sería caracterizado por Heidegger como el "paso atrás" (Schritt zurück) en textos como los cursos del Nietzsche y conferencias

28 SchürmanN, R. Des hégémonies brisées. Mauvezin, Trans-Europ-Repress, 1996, pp. 713, 770.

29 HeIDEgGer, M. Besinnung. Op. cit., p. 59: "(...) el espanto permanece la tonalidad fundamental, desde la que surge para ellos [los guardianes; die Wächter] la verdad de la sentencia: el Ser es la nada, cuyo rango esencial no alcanza poder alguno".

30 lb. p. 275. 
publicadas después de la guerra, es comentado en detalle en numerosos textos póstumos de la época. Su utilización subraya aquí que la historia del Ser es también y sobre todo una (otra) historia de la filosofía. Pero lo que nos parece determinante y queremos destacar es cómo Heidegger, pese a su radicalidad, se debate con un vocabulario tan conceptualmente cargado que no puede evitar recurrir a él para explicarse mejor, ya sea cuando habla de lo trascendental, del problema de las modalidades o incluso de la representación ${ }^{31}$. Podemos decir que, respecto al problema del nihilismo, y más concretamente respecto a la ausencia de fines propia de éste en la época moderna, asistimos a una situación semejante: ¿cómo hablar de fines que no caigan en el esquema tradicional?

No se trata, simplemente, de oponer a la obsesiva computación y al cálculo de fines fabricados una mera declaración de intenciones en las que se superen los fines como "metafísicos" y se abandonen sin más. La preparación e inauguración de otro pensar solo puede ser posible, a través de una decisión teñida por la experiencia del abandono del ser, si se logra la transformación en un nuevo pensar más allá de fines que bien podría semejar un nuevo y definitivo fin. Y en cierta medida, no se trata de otra cosa, tal y como expone Heidegger en unas de las consideraciones iniciales a las Contribuciones:

(...) la meditación inicial del pensar deviene necesariamente auténtico pensar, esto es, pensar que pone un fin (Ziel setzendes Denken). No es puesto cualquier fin y no el fin en general, sino el único y así singular fin de nuestra historia. Este fin es la búsqueda misma, la búsqueda del Ser ${ }^{32}$.

Hay aquí una inversión o aparente paradoja, entre el pensar que pone fines y la posición de fines que hemos caracterizado antes. ¿Cómo conjugar una afirmación semejante con los múltiples momentos en los que Heidegger opone este pensar, en una oposición aparentemente absoluta, a cualquier idea de "fin"? ¿No se afirma también en las Contribuciones que "el Ser (y por lo tanto la Nada) es el en-medio para el ente y la divinación (Götterung) y no puede ser nunca 'fin'"33? ¿Y a su vez, no se critican de nuevo en el §48 de La historia del Ser todos los intentos metafísicos que elijan entre trascendencia e inmanencia, "porque nunca plantean como fin el Ser" ${ }^{14}$ ?

31 Sobre el problema de lo trascendental en Heidegger, remitimos al interesante trabajo de GourdaIN, S. Sortir du transcendantal. Heidegger et sa lecture de Schelling. Bruselas, Ousia, 2018. Respecto a las modalidades, véanse en particular el artículo de MACDONALD, I. "Vers une démodalisation du possible. Heidegger et le clivage de l'estre", en Philosophies. № 140, 2019, pp. 21-30 y la sección "Le dépassement métaphysique du possible" de SERBAN, C. Phénoménologie de la possibilité. Husserl et Heidegger. Paría, Presses Universitaires de France, 2017, pp. 183-223.

32 Heidegger, M. Beiträge zur Philosophie (Vom Ereignis). Op. cit., p. 17.

33 lb. p. 267.

34 HeIDEgGer, M. Die Geschichte des Seyns. Op. cit., p. 55. 
Para resolver esta dificultad debemos entender que el cambio decisivo radica en que aquí la pretensión es que el pensar mismo, como pensar del Ser en su sentido productivo (Erdenken), sea el que "ponga" un fin, y que no dependa así de un fin ajeno como valor o idea que hubiera que alcanzar o cumplir. Ésa es justamente la perspectiva metafísica que, como ya hemos señalado, para Heidegger en estos años aboca a la autoaniquilación, y por eso mismo está aquí en juego la decisión entre historia o no-historia. Subrayemos además que por pensar productivo del Ser debemos entender el intento heideggeriano de un pensar que parte de y desde el Ser para interrogarlo como tal, previamente a toda caracterización o reducción metafísica. Más concretamente, no se puede hablar aquí, sin más, de algo externo al pensar del Ser; éste mismo no tiene un ámbito exterior y otro interior: si se parte desde el Ser es únicamente para pensar el Ser y no salir de su tematización, derivándola a partir de otros entes. Heidegger elabora este problema a partir de una retoma crítica de la noción de Inbegriffe, conceptos inclusivos, noción que ya había planteado antes, especialmente en el curso de 1929/30 sobre los Conceptos fundamentales de la metafísica, aunque en un sentido que se quería metafísico y englobante. Sin embargo, en el proyecto del pensar histórico del Ser, los Inbegriffe sirven para destacar más radicalmente cómo, dentro de "un saber que ya no es exterior a su 'objeto'", y por lo tanto no es exterior al Ser mismo, los conceptos se pertenecen mutuamente, señalados por el conjunto "inmanente" englobado por el In-: Innestehen, Inständigkeit, Innigkeit, etc ${ }^{35}$.

Todo esto nos conduce a entender mejor la respuesta que da Heidegger: que tan sólo este fin, tan sólo la búsqueda del Ser en cuanto tal, está en medida de aportar, o más bien de ser, una respuesta al nihilismo. Heidegger hace hincapié en esto en numerosas secciones de las Contribuciones, entre las que destacamos el $\S 38$, donde la búsqueda misma es caracterizada como "el encontrar originario"36. Aunque esta caracterización pueda resultarnos sorprendente por su simplicidad, nos hallamos aquí ante uno de los puntos decisivos del pensar histórico del Ser, e incluso, en cierto sentido, de una formulación clave del enigmático viraje o Kehre. A fin de cuentas, ¿qué puede ser una búsqueda que no parece poder ni deber concluir en encuentro alguno, en fin y final? Y precisamente, si nosotros como seres humanos no estamos en condiciones de responder mediante una decisión voluntaria y lanzarnos en la búsqueda del Ser, ¿cómo puede plantearse tal búsqueda?

35 DASTUR, F. Op. cit., p. 149; TRAWny, P. Op. cit., p. 9.

36 HeIDegger, M. Beiträge zur Philosophie (Vom Ereignis). Op. cit., p. 80. 
Para aclarar mejor este problema, un apartado de las "Reflexiones V", incluidas en el primer volumen de los Cuadernos negros, y por tanto contemporáneas de los textos aquí comentados, nos resulta de grandísima ayuda. Se trata del $\S 56$, donde Heidegger tematiza en mayor detalle el problema de la búsqueda y del fin, dirigiéndose especialmente a los alemanes, pero atañendo, no lo olvidemos, a toda posibilidad de humanidad futura.

En este apartado, Heidegger se pregunta directamente lo siguiente:

¿Pero y si el buscar fuera el más constante (beständigste) estar en la cercanía de lo que se oculta, desde lo cual nos acaece toda tribulación (Not) y nos espolea todo júbilo? iSi finalmente lográramos esta constancia, sin que reiteradamente se nos desintegrara a causa de la postura aparentemente afirmativa que incluso la mayoría de las veces pretende ser auténtica! ${ }^{37}$

No se trata, cabe mencionarlo aquí, de oponer al nihilismo la postura nietzscheana y afirmativa del amor fati. Para Heidegger, ésta no es la auténtica afirmación del decir-sí, propia, como veremos, del insistir del Da-sein, puesto que el amor fati se convierte en un mero consentir (zustimmen) al ente y no es otra cosa, en definitiva, que una continuación de la posición metafísica ${ }^{38}$.

La búsqueda debe elevarse entonces a fin, por paradójico que sea, del pensar del Ser, y en tal elevación, entendida la búsqueda como "sentido", "fin supremo" y "búsqueda de la verdad de la diferencia de Ser", Heidegger constata que el hombre logra no ponerse como "fin a sí mismo, sino aquella tonalidad fundamental de su esenciar como Da-sein..." ${ }^{\text {39. }}$ Así pues, como ya habíamos señalado, el pensar del Ser se opone a toda posición del hombre como fin, y por lo tanto a ese antropologismo metafísico de la época moderna, posición que concluye en la dominación de la fabricación como determinación de todas las experiencias, en tanto que vivencias dadas ahí, efectivamente, en su relación y medida para con el ser humano.

Es éste, además, uno de los sentidos insignes de la Kehre o viraje, tal y como precisa Heidegger a continuación:

Sí, pues aquí se anuncia el viraje, de modo que el hombre, si realmente es insistentemente (inständlich) el buscador, pertenece al viraje, y eso significa

37 HeIdegGeR, M. Überlegungen II-VI. (Schwärze Hefte 1931-1938). GA 94, Frankfurt am Main, Klostermann, 2014, p. 343.

38 HEIDEgGER, M. Besinnung. Op. cit., p. 120.

39 HeIDEgGER, M. Überlegungen II-VI. (Schwärze Hefte 1931-1938). Op. cit., p. 343. 
que el acontecimiento de hacer apropiado, en cuanto que esenciación

(Wesung) de la diferencia misma de ser, le ha prestado su adecuación ${ }^{40}$.

Si por viraje debemos entender el saltar fuera de lo trascendental y la tematización del Ser fuera de la distinción entre ser y ente, lo esencial aquí consiste en que no es el ser humano mismo el que comienza o lleva a cabo una acción, sino que es determinado a través del Ser-Ereignis y es marcado así por la diferencia ontológica entre ser y ente. Sin embargo, lo que resulta innegable es que el problema parece conducirnos, aunque sea a través de otras vías, ajenas a la metafísica, al papel que el ser humano puede y debe jugar en el otro comienzo.

Precisamente, en la sección de Meditación dedicada a la pregunta del ¿por qué? que ya hemos comentado, Heidegger da algunas indicaciones interesantes que profundizan lo expuesto hasta ahora, y completan cuestiones esbozadas en otros textos. Ahí subraya particularmente que el ¿por qué?, como pregunta originaria y transitoria, debe ser entendida bajo el prisma de la preparación (Vorbereitung) y del salto (Sprung). Ambos, preparación y salto, señalan en los textos de esta época el paso hacia otro comienzo del pensar, y por tanto también la superación del nihilismo y de la fabricación. En ese sentido, si la pregunta en su sentido auténtico nos conduce a lo inquietante que es la búsqueda del Ser, lo hace mediante una indicación fundamental, pues Heidegger apunta que la pregunta "nombra el claro, en el paso hacia el cual respectivamente el hombre transita la dignidad de la guardia de la verdad del Ser", y más concretamente, que si no se alcanza lo enigmático de la pregunta, se ingresa en "la alienación extrema respecto al Ser", como humanización (Vermenschung) total del ser humano ${ }^{41}$. Es decir, que el ser humano corre el riesgo de acabar encerrado en lo que ha sido o cómo ha sido comprendido metafísicamente: animal rationale, sujeto sometido y que somete el todo a la medida de lo fabricado, lo efectivo, lo producido, etc. Esto, dirá Heidegger, es nihilismo.

En el marco problemático que aquí estamos intentando clarificar, si el fin supremo del nuevo pensar es la búsqueda del Ser, queda entonces por precisar, aunque sea someramente, cómo una búsqueda tal puede ser mantenida, y más concretamente, cómo debemos mantenernos en esa búsqueda. Es en este punto donde surge con toda su fuerza la pregunta por el lugar del ser humano en relación con lo expuesto hasta el momento: ¿cómo podemos, nosotros, ser los buscadores del Ser? Este problema constituirá nuestro último apartado, en el que pretendemos exponer el papel del Da-sein en tanto que una respuesta a la superación del nihilismo en el final de la época moderna.

$40 \mathrm{lb}$.

41 HeIDEgGER, M. Besinnung. Op. cit., pp. 269-270. 


\subsection{La insistencia del Da-sein como buscador del Ser.}

El intento más radical de la historia del Ser, capaz de saltar más allá de la tematización conjunta del ser y del ente, consiste en desplazar el centro del preguntar desde el Dasein y su analítica existencial en Ser y tiempo a un pensar productivo del Ser, que no lo sitúe ni como punto de partida ni como destino final de toda la empresa metafísica. Pese a todo, cabe destacar que no se trata simplemente de dejar de lado al ser humano y pensar fuera de todo lo que le incumbe, sino más bien al contrario. Se trata de procurar acercarse de manera cada vez más decisiva, aunque sea por aparentes desvíos, a lo esencial: es decir, a través del Ser, repensar la humanitas de lo humano y no su reducción como "humanización metafísica"42, punto sobre el que Heidegger insistirá especialmente unos años después, en 1947, en su famosa carta a Jean Beaufret.

En ese sentido, si el pensar del Ser tiene como fin supremo la búsqueda del Ser en cuanto tal, la permanencia, el mantenimiento de esta búsqueda requiere al ser humano, y lo requiere, dirá Heidegger, no tal y como es o creemos que es, en sus determinaciones antropológicas y metafísicas, sino en tanto que éste es capaz de convertirse en Da-sein, albergando la posibilidad radical de realizarlo por medio de una experiencia fundamental

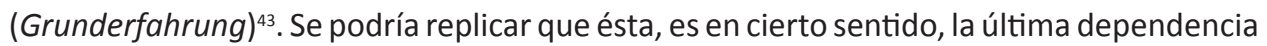
de los textos del Ereignis a una especie de marco trascendental que sin embargo pretenden dejar definitivamente atrás. Más allá de esta dependencia o superación, es innegable que la posibilidad del Da-sein (pues éste es ahora algo posible y desde luego no algo ya dado de antemano) es un tipo de condición de posibilidad respecto a la aprehensión del Ser en su esenciarse y en su verdad, y tiene una función, al menos, de copertenencia con el Ser en su búsqueda.

Respecto a este problema, y esto constituye uno de los puntos esenciales que tan sólo podemos esbozar aquí, pero al que nos consagramos en un trabajo de investigación de mayor envergadura, es que el Da-sein no es, estrictamente hablando, algo que concierna, tal cual, al ser humano en sus características presentes y efectivas. Pero, a la vez y paradójicamente, Heidegger da a entender en otros apartados que solo puede hablarse de Da-sein en relación al ser humano. A este respecto, la sección $§ 176$ de las Contribuciones aporta un esbozo de respuesta: el Da-sein pertenece al ser humano como su más alta posibilidad, y por tanto no sirve de nada nada precisar que es humano, como Heidegger afirma haber hecho en Ser y tiempo ${ }^{44}$. Lo relevante aquí es que el Da-sein

$42 \mathrm{lb}$.

43 HeIdegger, M. Beiträge zur Philosophie (Vom Ereignis). Op. cit., p. 61. 44 lb. pp. 300-301. 
no puede ser caracterizado a través de ninguna facultad o atributo alguno propios, o al menos tradicionalmente, del ser humano. Contra la metafísica y la fabricación que han culminado en el nihilismo, Heidegger denuncia en numerosos pasajes la elevación de tal o cual capacidad a rango superior de determinación del ente, como "hilo conductor y blanco de determinación del ente restante", desde la psyché y el anima, a las distintas figuras de la conciencia y de la voluntad pertenecientes al sujeto moderno y que concluyen en la radicalización del cuerpo y de los afectos en Nietzsche ${ }^{45}$. Se hace patente entonces que, para Heidegger, partir de un atributo del ser humano es ya entrar de lleno en la determinación metafísica del pensar a través del ente, y que este esquema de cosas no puede más que abocar en última instancia a la primacía de la vivencia tal y como el final de la época moderna atestigua.

En vez de este esquema de elevación, debemos (re)pensar el estatuto del Da-sein en tanto que buscador del Ser, como el que mantiene y se mantiene en esa búsqueda, siempre susceptible de recaer en una pérdida, como podría constituir la huida en la afirmación total y radical del ente. Esta única característica esencial, que podría entenderse como una radicalización de la comprensión del ser (Seinsverständnis) tematizada años antes, y pretende situarse fuera de toda comprensión antropológica de la metafísica, es la que Heidegger nombra in-sistencia o instancia, Inständigkeit. A través de esta definición, si aún podemos expresarnos así, nos referimos a algo que se pretende más sobre-humano que propiamente humano ${ }^{46}$. El Da-sein se convierte no tanto en una esencia inmutable de lo que es o lo que debe ser el ser humano, sino, atendiendo al sentido verbal de la esencia presente en estos textos, se trata de una posibilidad que puede esenciarse o desplegarse. Posibilidad que justamente solo puede corresponder al Ser mismo en su esenciar histórico y no en su reducción niveladora e indiferente como ser del ente, bajo el dominio de la fabricación. En ese sentido, cabe añadir que no es casual cómo el §38 de las Contribuciones, que ya hemos mencionado, concluya la exposición del buscar del Ser, determinado como encontrar originario, con las siguientes palabras: "dignificar lo más digno de cuestión, perseverar en el preguntar, insistencia (Inständlichkeit)"47.

\footnotetext{
$45 \mathrm{lb}$. pp. 312-313.
}

46 HEIDEGGER, M. Zu eigenen Veröffentlichungen. GA 82. Frankfurt am Main, Klostermann, 2018, p. 24. Así es como Heidegger caracteriza al Da-sein en el volumen 82, en una serie de comentarios a Ser y tiempo escritos en torno a la misma época, donde podemos apreciar la evolución de gran parte de sus conceptos en el viraje hacia el pensar histórico del Ser. Para un acercamiento a estos comentarios, aún pendientes de ser explorados en detalle, remitimos a VERAZA, P. "La transformación conjunta del Da-sein y el método en las Laufende Anmerkungen de Heidegger", en Apeirón. № 135, 2018, pp. 135-145.

47 Heidegger, M. Beiträge zur Philosophie (Vom Ereignis). Op. cit., p. 80. 
Para precisar y profundizar el sentido y la dimensión de la in-sistencia, y volviendo a la caracterización que da Heidegger en el denso apartado de Meditación sobre la pregunta del ¿por qué? (del que nunca hemos salido del todo), encontramos lo siguiente:

El Da-sein, en el que el hombre transformado deviene insistente (inständig), se mantiene (hält sich) en la abismosa cercanía al Ser; sin embargo este mantenerse (Sichhalten) nunca puede devenir para el hombre un estado duradero habitual, que transcurra por sí, sino que se esencia en la resolución (Entschlossenheit) ${ }^{48}$.

En tanto que posibilidad que debe mantenerse en la insistencia, el Da-sein es transitorio y fugaz, una especie de destello que, en un instante, surge tan repentinamente como se apaga. Pero esto es necesario y esencial, y es en esta cercanía al abismo del Ser que vemos un eco de esa incapacidad metafísica que el filósofo alemán quiere superar: el nihilismo. Esencialmente incapaz de pensar el Ser, así como la nada en el Ser mismo, el nihilismo no se podrá afrontar a través del sujeto moderno sino con su última transformación en Da-sein, asumiéndose a través de la resolución, ya que "jamás por el mero esfuerzo de la voluntad del hombre (...) el abrirse que cuestiona-se atreve al golpe (Sto6) del Ser"49.

Es, ciertamente, una posibilidad extraña y enigmática dentro del marco de la historia del Ser, pero sin la cual esta historia no puede ser aprehendida en toda su envergadura. Pues, justamente, es sólo por la in-sistencia del Da-sein, como señala Heidegger en el $\S 193$ de las Contribuciones, que podemos alcanzar un tipo de saber no-metafísico: "nuestro saber (Wissen) alcanza solo en la medida en que se extiende la in-sistencia en el Da-sein"50. Es este saber, que se revela más humilde y alejado de todo tipo de dominación, ajeno a todo conocimiento establecido a través de la certeza, de la ciencia y del aseguramiento metafísico del ente, el que aspira a alcanzar el pensar del Ser, en tanto que verdad del Ser. Pero al alejarse justamente del eterno moverse en el marco del ente y de su dominio, Heidegger puede caracterizar en La historia del ser este tipo de saber, propio de la in-sistencia, como el "saber que actúa más que toda acción al servicio de la fabricación, puesto que su esencia no exige ningún resultado, solamente es, lo que es, en la medida que es"51. Todo ello nos conduce a afirmar que el saber de la in-sistencia es el que permite, a través de la decisión y de la resolución, vislumbrar la posibilidad de una historia auténtica, dejando atrás la repetición constante de la época moderna en

48 HeIDEgGER, M. Besinnung. Op. cit., p. 275.

$49 \mathrm{lb}$.

50 Heidegger, M. Beiträge zur Philosophie (Vom Ereignis). Op. cit., p. 375.

51 HeIDEGger, M. Die Geschichte des Seyns. Op. cit., p. 197. 
la que culmina la metafísica: la fabricación y la reducción del todo a fines vacíos, en un retroalimentarse indefinido de vivencias inesenciales. Es decir, en nihilismo.

En la medida en que se despliega como buscador del Ser en la in-sistencia, el ser humano hace la experiencia fundamental de transformarse en Da-sein. Este saber, a través de esta experiencia, es precisamente el que Heidegger caracteriza de manera casi nietzscheana en el $§ 72$ de las Contribuciones como saber del nihilismo, como "lo más intransitable y pesado"52. Más allá de la ausencia de fines, aquí se abre la vía a otra de las dimensiones de superación del nihilismo, y, co-perteneciéndole, a otra de las confrontaciones más esenciales y radicales del pensar histórico del Ser contra la metafísica, y contra Nietzsche en particular. Como subraya Heidegger en esa página: "en la experiencia fundamental, en la que el hombre como fundador del Da-sein es requerido por la divinidad del otro dios, se inicia la preparación de la superación del nihilismo"53.

Nos gustaría concluir con esta indicación, con una señal que apunta a la llegada de ese otro y último dios, posibilidad última que marca la repetición y la superación de dos de los más imponentes dicta de Nietzsche: la muerte de Dios y, tal y como se lamenta en el $\S 19$ de El Anticristo, la urgente necesidad de crear nuevos dioses tras casi dos milenios de cristianismo. Si Dios ha muerto, si éste es uno de los acontecimientos fundamentales de la historia de la metafísica y de su final, no debemos olvidar que aquel que lo anuncia, der tolle Mensch, es también aquel que busca a dios, y así es presentado por Heidegger en las primeras páginas de La esencia del nihilismo: como el "buscador de Dios" (Gottsucher) ${ }^{54}$. Si el nihilismo, entendido como pérdida de sentido y ausencia de todos los fines, tan solo puede ser superado por el pensar que se mantiene en la búsqueda del Ser, en tanto que fin supremo más allá de la metafísica, con esta aproximación se nos abre un nuevo problema. Nos falta aún por determinar qué significa exactamente que el ser humano, a través de su transformación en Da-sein, sea también un nuevo buscador de dios, y cómo es requerido por este último. Solo así entenderemos, en toda su plenitud, cómo el pensar histórico del Ser constituye la respuesta decisiva a la pregunta ¿por qué?

52 HeIdegGer, M. Beiträge zur Philosophie (Vom Ereignis). Op. cit., pp. 140-141.

$53 \mathrm{lb}$.

54 Heidegger, M. Metaphysik und Nihilismus. Op. cit., pp. 193-194. El texto está incluido como segunda parte del volumen 67 , y recoge en su mayoría páginas ya publicadas en el segundo volumen del Nietzsche, salvo las primeras, donde precisamente se menciona al Gottsucher. 


\section{Referencias bibliográficas.}

Alvarado, Á. "Consideraciones sobre la raza, el cuerpo y el mestizaje a la luz de los Cuadernos negros de Heidegger", en Eikasia. № 91, 2020, pp. 37-58.

DASTUR, F. "Le tournant de l'Ereignis et la pensée à venir", en SCHNELL, A. (Ed.). Lire les Beiträge zur Philosophie. París, Hermann, 2017, pp. 141-158.

Gourdain, S. Sortir du transcendantal. Heidegger et sa lecture de Schelling. Bruselas, Ousia, 2018.

HeIdegger, M. Beiträge zur Philosophie. (Vom Ereignis). GA 65. Frankfurt am Main, Klostermann, 1989 (Aportes a la filosofía (Acerca del evento). Tr. D. Picotti. Buenos Aires, Biblos, 2003).

HeIDEgGeR, M. Besinnung. GA 66. Frankfurt am Main, Klostermann, 1997 (Meditación. Tr. D. Picotti. Buenos Aires, Biblos, 2006); (Méditation. Tr. A. Boutot. París, Gallimard, 2019). HeIDEgGeR, M. Des Geschichte des Seyns. GA 69. Frankfurt am Main, Klostermann, 1998 (La historia del ser. Tr. D. Picotti. Buenos Aires, El hilo de Ariadna, 2003).

HeIDEGger, M. Metaphysik und Nihilismus. GA 67. Frankfurt am Main, Klostermann, 1999. HeIDEgGeR, M. Überlegungen II-VI. (Schwärze Hefte 1931-1938). GA 94. Frankfurt am Main, Klostermann, 2014 (Cuadernos negros 1931-1938. Tr. A. Ciria. Madrid, Trotta, 2017).

HEIDEGGER, M. Zu eigenen Veröffentlichungen. GA 82. Frankfurt am Main, Klostermann, 2018.

MAcdonald, I. "Vers une démodalisation du possible. Heidegger et le clivage de l'estre", en Philosophie. № 140, 2019, pp. 21-30.

Polt, R. The Emergency of Being. On Heidegger's "Contributions to Philosophy". Ithaca, Cornell University Press, 2006.

SchüRmAnN, R. Des hégémonies brisées. Mauvezin, Trans-Europ-Repress, 1996.

Serban, C. Phénoménologie de la possibilité. Husserl et Heidegger. París, Presses Universitaires de France, 2017.

SeVerino, E. Esencia del nihilismo. Madrid, Taurus, 1991.

SOMmer, C. "Für die Wenigen - Für die Seltenen. Doctrine esotérique et art d'écrire chez Heidegger", en SCHnelL, A. (Ed.). Lire les Beiträge zur Philosophie de Heidegger. París, Hermann, 2017, pp. 7-24. 
SOMmer, C. Mythologie de l'événement. Heidegger avec Hölderlin. París, Presses Universitaires de France, 2017.

Trawny, P. Adyton. Heideggers esoterische Philosophie. Berlín, M\&S, 2010.

VerAzA, P. "La transformación conjunta del Da-sein y el método en las Laufende Anmerkungen de Heidegger", en Apeirón. № 135, 2018, pp. 135-145.

Xolocotzı, Á., "De la metafísica del Dasein a la metapolítica del pueblo histórico. Aspectos políticos del giro de Heidegger", en O que nos faz pensar. № 36, 2015, pp. 7-25.

XolocotZı, Á. Fundamento y abismo. Aproximaciones al Heidegger tardío. México D. F., Porrúa, 2011.

Xolocotzi, Á.; Gibu, R.; Huerta, V. y VerazA, P. Heidegger. Del sentido a la historia. México D. F., Plaza y Valdés, 2014. 